\title{
An Effective Route Selection Scheme with Considering Traffic Density in VANET
}

\author{
Do-Sik An, Gi-Hwan Cho, Member, KIMICS
}

\begin{abstract}
A geographical routing protocol is typically utilized for a route selection of VANET. Even if it is conceptually well adapted into VANET, message delivery tends to be unreliable with frequent network partitions, which mainly come from the inherent characteristics such as high mobility and irregular traffic density. In this paper, we deal with a method to enable reliable message delivery with reflecting the traffic density on routing decision. By concatenating the message delivery cost of each of inbetween junction section, $1^{\text {st }}$ and $2^{\text {nd }}$ shortest paths are initially determined. When it is not possible to deliver the message on a junction on the $1^{\text {st }}$ path, we utilize two novel ideas, that is, letting the message stay on the junction to find out a new relay node, and putting a replicated copy to reach via a detour path. By using the NS-2 network simulator, our method has been examined in terms of message delivery rate and delay. It shows that our method is much efficient than the other method in the low density environment, while it brings similar results in the high density environment.
\end{abstract}

Index Terms-VANET, Route Selection, Geographical Routing, Traffic Density.

\section{I.INTRODUCTION}

Vehicle-to-Vehicle communication is getting to interest from the modern information and communication societies. It is beneficial in providing intelligent transportation system as well as driver's and passenger's assistant services. In this context, Vehicular Ad hoc NETwork (VANET) is a form of self- organizing wireless ad hoc network, which enables to communicate between the moving vehicles without supporting an infrastructure. It basically aims to make a vehicle notify some useful information, such as road condition and traffic situation, to neighboring or following vehicles. With this feature, some degree of vehicular and/or driver safety can be achieved by means of collision avoidance, cooperating derive, dangerous event warning [1],[2]. Emerging VANET applications often make use of wide-area coverage, so they usually require multi-hop routing

Manuscript received October 29, 2010;revised November 11, 2010; accepted November 18, 2010.

Do-SikAn is with the Division of Electronics and Information, Chonbuk National University, Jeonju, Korea (Email: rokmcads@jbnu.ac.kr)

Gi-Hwan Cho is with the Division of Computer Science and Engineering, Chonbuk National University, Jeonju, Korea (Email: ghcho@jbnu.ac.kr) * correspondent author protocols. For the sake of effective routing, the protocol design should take account into the VANET's inherent features.

VANET can be mainly characterized from Mobile Ad hoc NETwork (MANET), in terms of frequent topology changes by dynamically moving vehicles. So it usually makes use of a geographical routing method rather than well-known MANET routing protocols, as AODV, OSLR, and DSR [3]. As a typical geographical routing protocol, GPSR [4] is trying to forward message in greedy to the nodes toward the destination. Thus, it chooses the node close to the destination as a reply node for the sake of effective routing. When the greedy forwarding is not possible any more (so, local maximum), a recovery mode is taken part in to resolve this trouble. It tries to make use of a detour route to eventually deliver a message to the destination.

Even if the geographical routing is conceptually well adapted into VANET, it brings about frequent recovery modes in the complicated road/traffic environment. Especially, in the low densely deployed environment, frequent network partitions are inevitable with vehicle's high mobility and irregular traffic density. Then, the message will be delivered with long time trip due to the frequent recovery mode. Therefore, it is very important to device a method to convert this situation in order to enable VANET in practice [5]-[7].

On the one hand, most modern cities provide traffic information as node density, through Vehicle Detection System (VDS). If this traffic information can be utilized to establish a routing path, messages might be delivered to the destination in high reliability [8]. That is why we are trying to adapt the traffic situation to VANET routing.

This paper deals with an effective route selection method in the VANET environment. Firstly, we adapt the current traffic situation into routing decision. By concatenating the message delivery cost which reflects the traffic density of each in-between junction as well as the distance from the source to the destination, shortest paths are initially determined. Secondary, if it is not possible to deliver the message on a junction on the $1^{\text {st }}$ shortest path, we make use of two ideas to resolve this issue; letting the message stay on the junction to find out a new relay node, and putting a replicated copy to reach via a detour path. With benefit of these special treatments, we can expect to increase not only the rate passing through on the shortest path, but also the message delivery rate. 


\section{RELATED WORK}

\section{A. GPSR routing protocol}

GPSR protocol which is one of position-based protocols forwards a packet to a neighbor node which is geographically closer to the destination node among the neighboring nodes. This kind of routing protocol is named as greedy forwarding. To select a relay node, the positional information of the neighbor node has to be known by using a periodic beacon message. Each node can know the own location making use of device such as GPS, and also find a neighbor's location by exchange the Hello beacon message.

GPSR tries to forward a message with the greedy mode and recovery mode to the destination node. The greedy mode selects a relay node as the greed forwarding pattern.In the greedy mode selection, if a node confronts with the local maximum situation where it cannot discover the relay node among adjacent nodes which is closer to the destination node than itself, it changes its operation mode into the recovery mode.

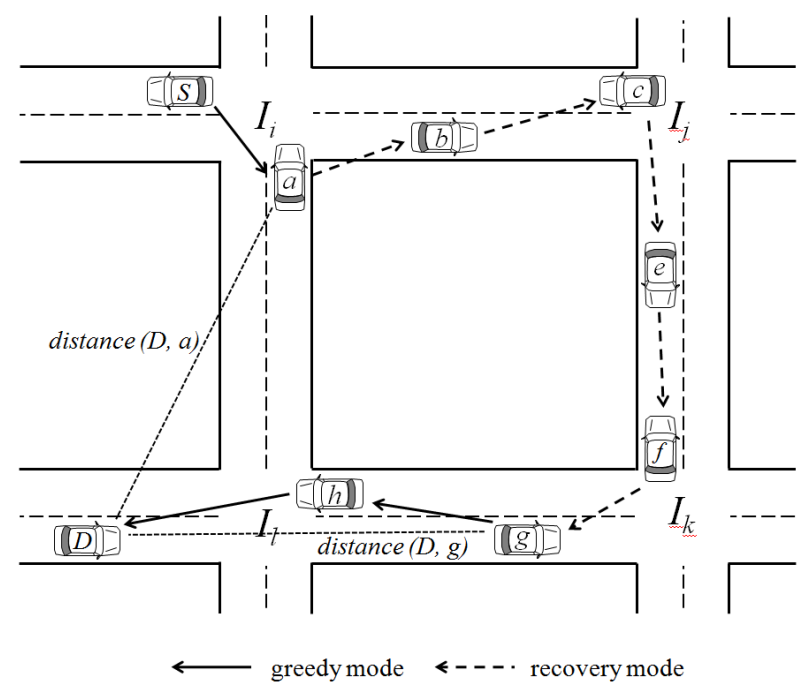

Fig. 1. An example of GPSR routing.

Fig. 1 shows the example of GPSR routing. By using the greedy mode, the node $S$ transmits a message to the node $a$. The node $a$ is confronted with the local maximum situation where it cannot discover the relay node which is closer to the destination node. As the recovery mode, the node $a$ recordsits own location in the packet header and transmits the message to the node $b$ based on the right-hand rule. On receiving the message, the node $c$ calculates the distance between itself and the destination node, and then compares it with the distance between node $b$ and the destination node. Because the node $c$ is much close to the destination, it goes to forward the message further. Whenthe message arrives at the node $g$ with the recovery mode, the distance $(D, g)$ is getting shorter than the distance $(D, a)$, so the routing mode is changed into the greedy mode.

\section{B. GPSR routing failure in the urban environment}

In the low density VANET environment, it is well known that GPSR's routing efficiency is quite low because it cannot select a reply node in frequent[9]. Especially, this characteristic inevitably brings about a network partition, then routing might be failed to forward messages any more [3],[5].

In the traffic road environment, it is common to have a lot of alternative paths between the given two points. In addition, vehicles would be quickly appeared or disappeared at a given area. However, GPSR considers the static geographical topology, and tries to repeatedly find out an alternate path at the routing failed point (that is recovery mode). Moreover it does not take care of current traffic situation and vehicle node's dynamics [10],[11].

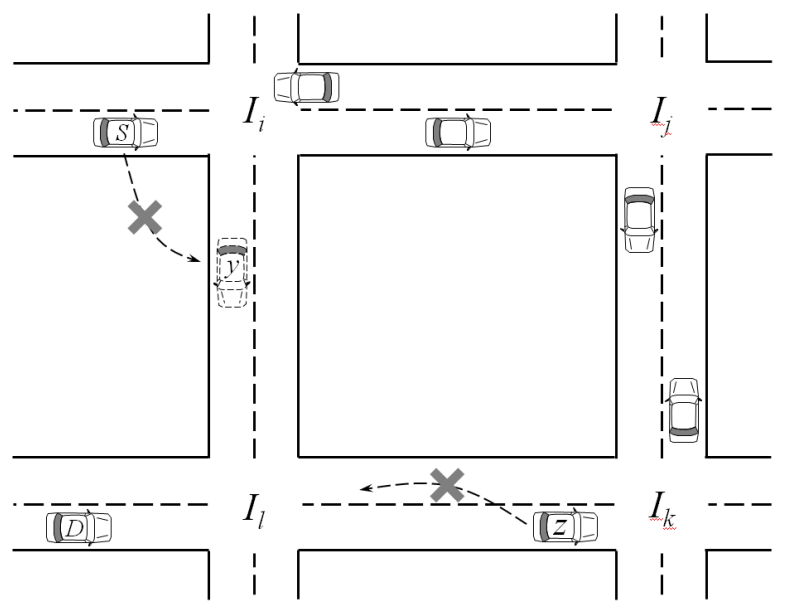

Fig. 2. An example of GPSR routing failure situation.

Fig. 2 shows an example of GPSR routing failure in the urban environment. Initially, the source node $S$ sends a message to the neighbor node which is close to the destination $D$. When the message arrives at the intersection $I_{i}$, GPSR has to change its operation into the recovery mode because there is no vehicle toward the destination. This situation is the same at the intersection $I_{k}$. If GPSR protocol cannot find out any relay node at this point, it would be eventually failed to forward the message any more.

\section{THE ROUTING PATH DECISION WITH CONSIDERING TRAFFIC DENSITY}

\section{A. Traffic density}

In the modern information and communication infrastructure, traffic density can be easily obtained by means of VDS. By detecting vehicles with loop detector and/or camera, VDS provides some useful data, such as traffic volume, vehicle speed, and 
occupation time. It is usually deployed and operated in highway and main road in city. The collected data are aggregated into the traffic control center. Along with some degree of processing the data, the traffic density information is delivered to drivers in order to be utilized to choose their own route.

One possible way to get the traffic density is to make use of the vehicle's occupation time. This can be represented as below [12].

$$
\text { vehicle density }=
$$

Thus,

$T \quad$ selected time period

$N \quad$ number of vehicles detected in time period $T$ by VDS

$t_{\text {occ }}$ time that the selected detector is turned on

The vehicle density informally stands for how many vehicles passed through VDS for a giventime period $T$.In a typical VDS system such as loop detector, the time to detect a vehicle is modeled as the time on which VDS tunes. Therefore,the vehicle density can be calculated by dividingthe time on which VDS tunes into the time period T.Fig. 3 shows an example to measure the vehicle density using VDS. It is assumed that each VDS tries to sense vehicles lane by lane.

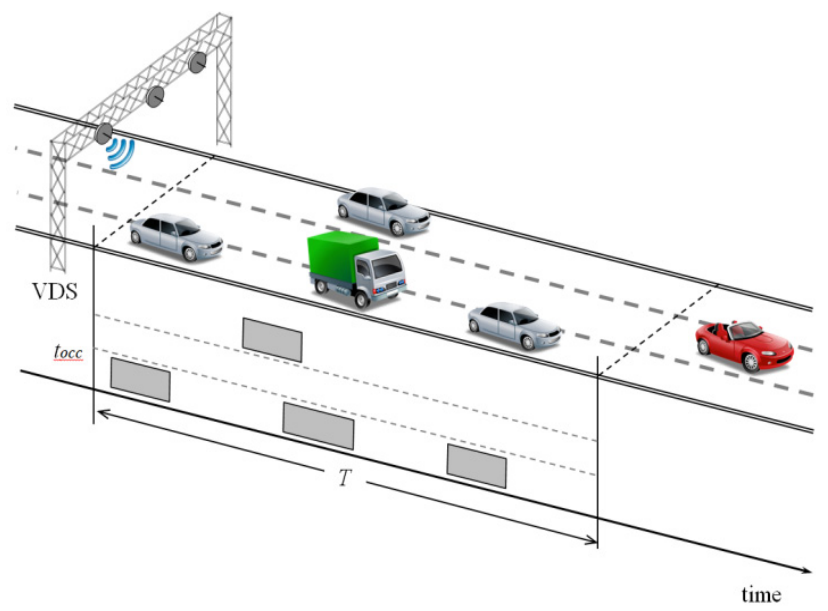

Fig. 3. An example of VDS.

\section{B. The shortest path considering traffic density}

It is common that the vehicle node density is usually not even in the different regions of the urban environment. Thus, if messages are routed based on only the distance to destination, it will bring about frequent routing failures and/or long delay time in the low density region. Therefore, it is clear that the road density plays great role in the reliable and/or fast message delivery. The road density can be obtained with the formula (1).

The shortest path between the source and the destination can be calculated on concatenating each expected message delivery cost of in-between junction section. An expected message delivery cost of each section can be obtained by weighted multiplexing the number of hops into the vehicle node density. Formula (2) shows one possible way to get the message delivery cost.

$$
e c_{i j}=\left\lceil\frac{d_{i j}}{r}\right\rceil \times\left(1-\frac{\sum_{n=1}^{N D_{i j}} \frac{\sum_{n=1}^{N V} t_{o c c}}{T}}{N D_{i j}}\right) \times a(2)
$$

Thus,

$e c_{i j} \quad$ expected packet forwarding cost from $I_{i}$ to $I_{j}$

$d_{i j} \quad$ distance from $I_{i}$ to $I_{j}$

$r \quad$ communication range

$N V$ the number of vehicles detected in time duration $T$

$N D_{i j}$ the number of detectors from $I_{i}$ to $I_{j}$

A correction factor

For the sake of flexibility and availability, it is sometimes much better to make use of an alternative path, like as $1^{\text {st }}$ shortest path and $2^{\text {nd }}$ shortest path (sometimes this is called as an alternative path) [13].

\section{IV.THE ROUTE SELECTION METHOD}

To begin with, we assume that the sender can overhear the message sent by the receiver. This permits the sender to confirm whether the message sent just before has been received by the receiver, and even which node (including its location) has received. Our route selection method consists of two operation modes; straight mode and intersection mode. If messages are delivered to a relay node on $1^{\text {st }}$ shortest path, our method operates in the straight mode. When the method confronts a condition which cannot be forwarded any more, it changes its operation into the intersection mode. Here, two ideas are taken part in reliable message delivery as described below. 


\section{A. Straight mode}

The straight mode stands for an initial and normal operation. In other word, whenever the sender can make sure that the message sent by itself has been delivered to a relay node located on $1^{\text {st }}$ shortest path, the route selection works in straight mode. In this mode, messages are basically forwarded in greedy. That is, the closestnode from the current location to the destination on the shortest path is selected as the next relay node.

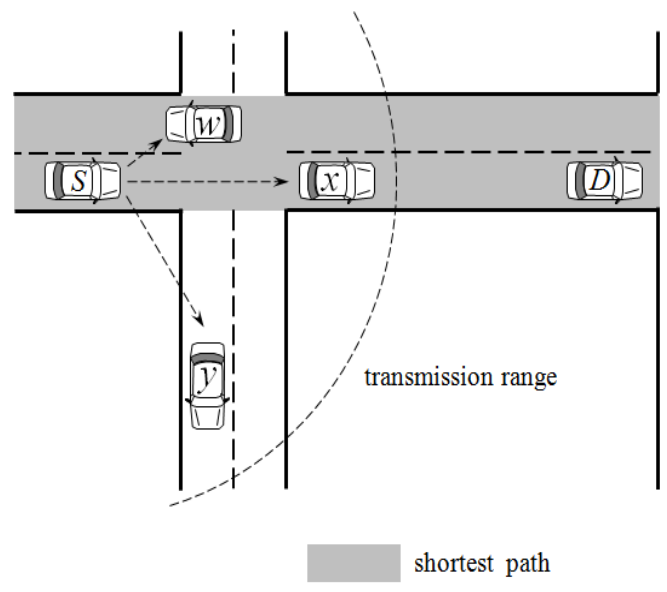

Fig. 4. An example of the relay node selection.

Fig. 4 shows an example of the relay node selection in the straight mode. Initially, the source node Scalculates the shortest paths $\left(1^{\text {st }}\right.$ and $\left.2^{\text {nd }}\right)$ to the destinationwith considering the traffic density. It then sends a message which includes the shortest paths information. The message is delivered to all neighbor nodes within the radio coverage of the sender. Each of neighbor nodes decides whether it is on $1^{\text {st }}$ shortest path, which can be extracted from the received message. In the figure, the neighbor nodes on $1^{\text {st }}$ shortest path, that is, $w$ and $x$ become the candidates of next relay node. But, the node $y$ would give up to be the next relay node because it is not located on $1^{\text {st }}$ shortest path.

Each relay candidate node calculates the distance on the shortest path from itself to the destination. Then, it sets up its own timer based on the distance; the node with longer distance may set the timer longer. In the figure, the node $x$ may set its timer short because it is located the most closely to the destination, while the node wmay set its timer long. The node which has the shortest defer-time starts to broadcast the message. This actually plays a role of the other means to notify itself as the next relay node. Then, the other candidate nodes give up to be the next relay node because they received the same message at least twice; that is, two messages comes from the source and the node $x$.
On receiving the message from node $x$, the source node can confirm that the message it sent has been safely forwarded to the next relay node. With this condition, each sender can confirm its reliable message delivery. If the source does not receive the next relayed message within a pre-defined time, it has to try to re-send the message for the reliable delivery.

\section{B. Intersection mode}

If the source or a relay node finds out that it cannot forward the message on $1^{\text {st }}$ shortest path anymore, it changes its operation state from the straight mode to the intersection mode. This mode is mainly taken place in the intersection region. Here, we define the 1.5 times area of wireless coverage from the intersection center as an intersection region. Two ideas are utilized to resolve this situation; first approach is to let the message stay on the junction to find out a new relay node, and second one is to put a replicated copy to reach via a detour path. The operational details of them will be given below.

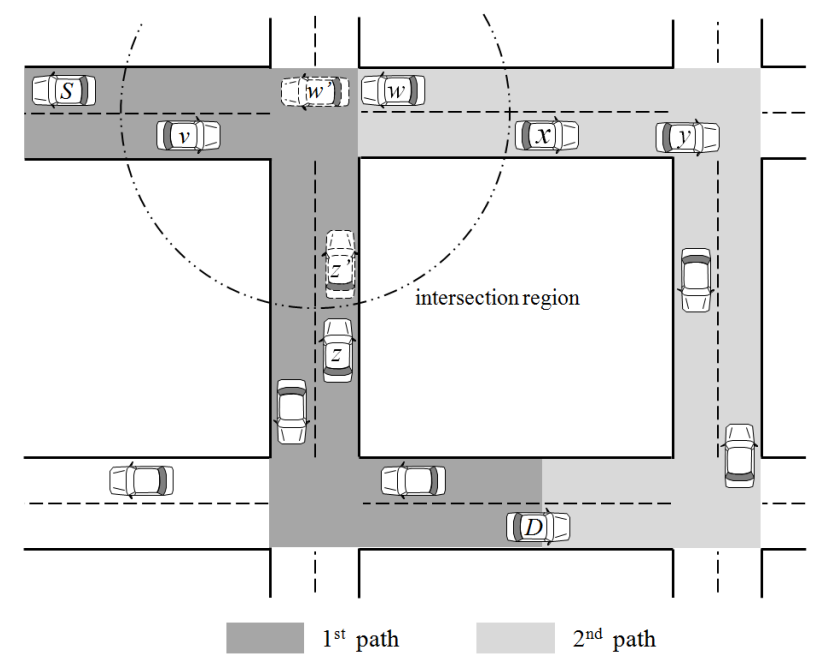

Fig. 5. An example operation of the intersection mode.

Fig. 5 shows an operational example of the intersection mode. Here, $w^{\prime}$ and $z^{\prime}$ stand for new location some time later of $w$ and $z$ respectively. Initially, let's assume the node $v$ has been selected as the relay node for the message sent by the source node $S$, using the straight mode. When the node $v$ tries to forward a message to the next relay node, only the node $w$ will receive the message because it is only the node within the coverage of node $v$. Repeatedly, after the node $w$ broadcasts the message, if it does not overhear any transmission except the node $v$ which is just the previous relay node, it finds out that the message cannot be forwarded through the $1^{\text {st }}$ shortest path. Then, the node $w$ changes its operation state into the intersection mode. 
Surely, if the node $w$ has been heard its forwarded message from the neighbor, node $z^{\prime}$, the message will be kept to be forwarded with the straight mode. On the other hand, it heard its forwarded message from the other neighbor, node $x$, the node $w$ will keep trying to forward with the intersection mode because node $x$ is out of $1^{\text {st }}$ shortest path. If the next relay node, node $x$, is located out of the intersection region, the sender, thus, $w$, keep the original message with the intersection mode, while the node $x$ tries to forward a replicated message through $2^{\text {nd }}$ path with the straight mode. So, the replicated message will be forwarded in the straight mode to the node $y$. It would finally deliver to the destination, or it is possible to fail to relay the message.

Thus, if a relay node within the intersection mode cannot overheard the message it sent, it will try to send the message repeatedly, practically for each $100 \mathrm{~ms}$. Then, a node approaching to the current relay node will take the forwarded message, and tries to keep on delivering the message with the intersection mode. When the node eventually finds out that the message sent to a vehicle destined to $1^{\text {st }}$ path, it stops to pass the message with intersection mode. This idea works much like to stay on the message in the intersection region.

With repeatedly re-sending feature until the sender success to deliver a message to the next relay node, it is reasonable to limit the number of retry to forward the message in the intersection region. Without this limitation, the message can be endlessly stay on the intersection region. If a relay node within the intersection region fails to forward the message into $1^{\text {st }}$ shortest path until it gets out from the area, it will give up to become a relay node. Here, if the replicated message also was failed to be forwarded to the destination, this message delivery would be eventually failed.

\section{PERFORMANCE EVALUATION}

In this section, we show some results of performance evaluation for our route selection method by comparing with GPSR protocol. Recall that our work basically focuses on improving the packet deliver rate. For the evaluation, we suggest a simulation model, and then show a couple of simulation results. Here, the vehicle movement model is assumed to be the Manhattan model. A simulation has been conducted with the network simulator, NS-2(2.33) [14].

\section{A. Simulation environment}

In this simulation, we assume that all nodes know their own location information through Global Positioning System (GPS) like tool. The location of an intersection and a road can be recognized by using a digital map. In our experiment, we assume that a vehicle moves around the roads which are arranged in $3,000 \mathrm{~m} \times 3,000 \mathrm{~m}$ driving field. Fig. 6 shows the simulation topology. Each car moves the roads which are apart $600 \mathrm{~m}$ each other. It's moving speed and direction is modeled with Manhattan model.

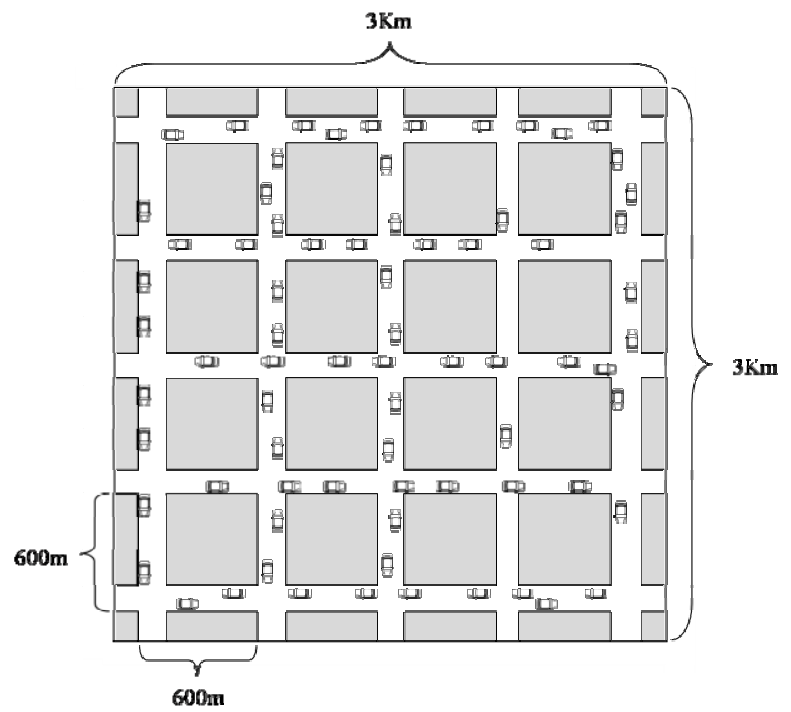

Fig. 6. The simulation model.

Table 1 indicates the parameters used in our simulation. The number of vehicles which take part in the simulation is $40 \sim 140$. Our work aims to take a performance comparison according to change the number of vehicles. Vehicles move with the in-between speeds $40 \sim 100 \mathrm{Km} / \mathrm{h}$, and they change their speed in periodically.

We assume the communication scenario used for the simulation as fellow. The packet size is 512 bytes. In addition, the communication range is set as $250 \mathrm{~m}$. IEEE 802.11 MAC is used for data transfer protocol because the network interface for VANET was designed based on IEEE 802.11 in the standardization body. For the GPSR protocol, it is assume that it transmits the beacon message in one second interval in order to collect the neighbor node information. The simulation time has been performed for 200 seconds.

TABLE I

SIMULATION PARAMETERS

\begin{tabular}{|l|l|}
\hline \multicolumn{1}{|c|}{ parameter } & \multicolumn{1}{c|}{ value } \\
\hline number of vehicles & $40 \sim 140$ nodes \\
\hline velocity of vehicle & $40 \sim 100 \mathrm{Km} / \mathrm{h}$ \\
\hline wireless communication range & $250 \mathrm{~m}$ \\
\hline MAC protocol & IEEE $802.11 \mathrm{MAC}$ \\
\hline packet size & 512 byte \\
\hline Hellobeacon period (GPSR) & 1 second \\
\hline simulation time & 200 seconds \\
\hline
\end{tabular}




\section{B. Simulation result}

The simulation has been repeated 5 times with the same parameters, and then calculated an average of the results. The packet delivery rate, packet delay time, and packet delivery hops have been evaluated according to change the number of vehicle nodes.

Fig. 7 shows the packet delivery rate over different node density. In the case of GPSR, when the number of nodes is below 60 , the message delivery rate is less than $5 \%$. As to the proposed method, because of transmitting a packet with the route where the node density is high, the delivery rate was improved about $10 \%$ in comparison with GPSR.

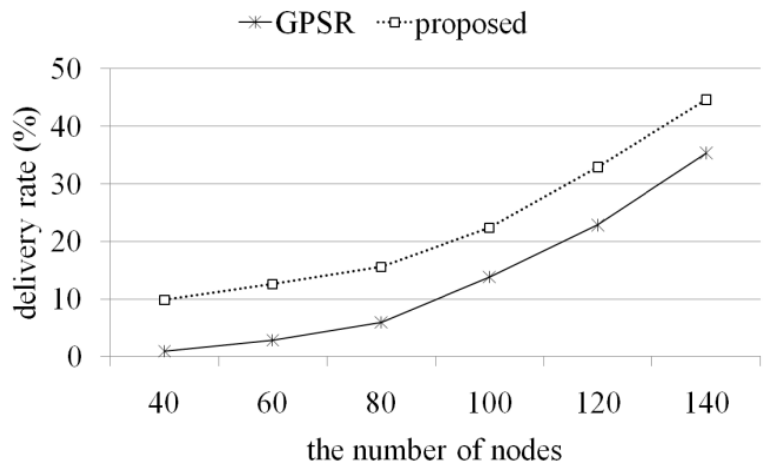

Fig. 7. Packet delivery rate.

Fig. 8 shows the end-to-end average packet delivery delay time over different node density. In the Fig. 7, two methods show quite different packet delivery rate for all range of node density. However, when the node density is low, the end-to-end packet delivery delay time of proposed method is bigger than that of GPSR, while as the number of node is increased, the delay time is getting to similar to GPSR.

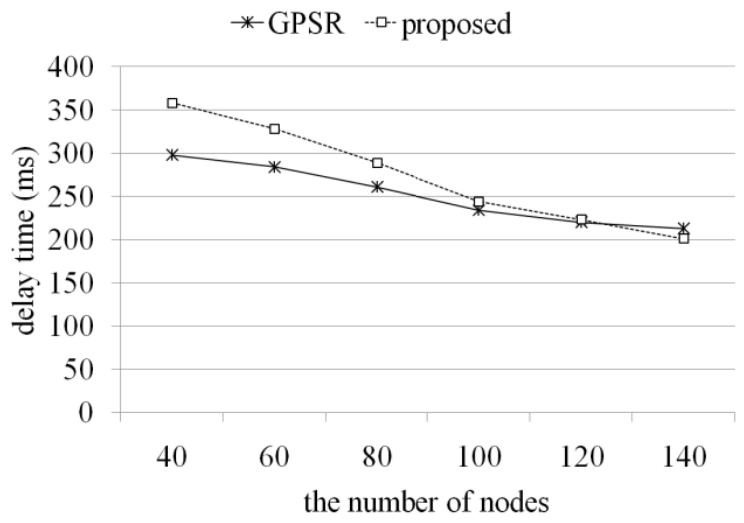

Fig. 8. End to end delay.

Fig. 9 shows the maximum packet transfer delay time for a single hop delivery. In a situation where the node density is low, the difference of packet delay time was big much like as the end- to-end delivery delay time. But, the proposed method has much bigger delay time than GPSR in single hop. If the node number is increased, the delay time is getting to similar to GPSR. For the proposed method, because it utilizes the vehicle density and transmits a packet on the path with high density, the delay time for a single-hop delivery is getting to close to GPSR.

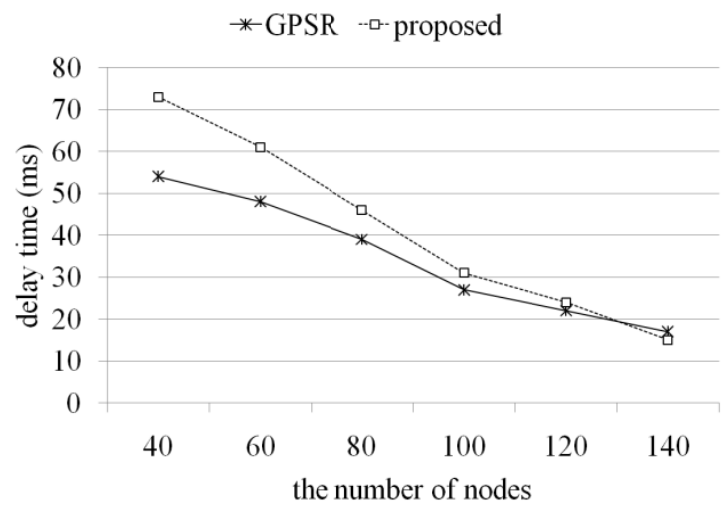

Fig. 9. Maximum delay in single-hop delivery.

Fig. 10 is the simulation result of the average hop number for end-to-end packet delivery. The proposed method has small hops in compared with GPSR when the node density is low. This comes from the fact that the proposed method tries to re-send a packet if there is no relay node. However, with participating about 100 nodes, the proposed method shows that the number of hops is getting bigger than GPSR because it tries to send a packet to the $1^{\text {st }}$ path in on intersection mode. Then, the number of hops is similar to GPSR on increasing the node density.

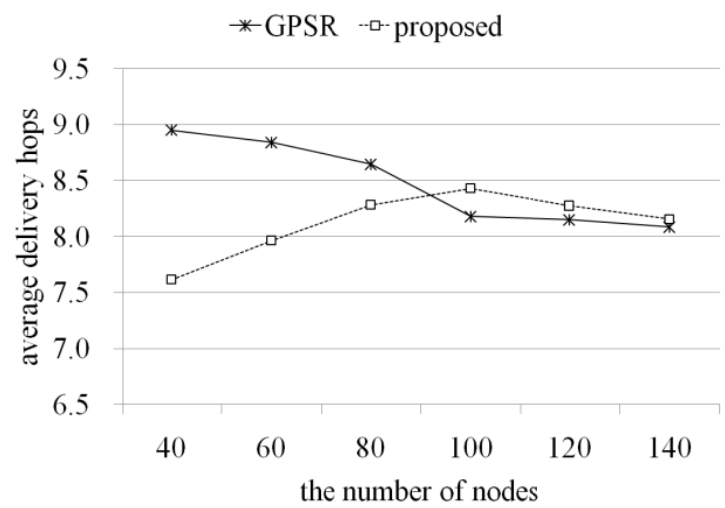

Fig. 10. Average hop number.

The proposed method shows certain degree of performance improvement on message delivery rate in the low vehicle density environment, while it takes much higher delay time than GPSR. However, in the high density environment, while the message delivery rate is kept high, the delay time is getting to similar with that of GPSR. In summary, we argue that it is very important to calculate the message delivery path selection with considering the newest traffic situation. 


\section{CONCLUSIONS}

In order to enable an effective route selection as well as a reliable message delivery in VANET environment, this paper includes three main ideas; making use of traffic situation on route decision, applying a duplicated message copy for alternative routing, and adapting message stay effect in the intersection region to increase reliability. With reflecting not only the distance but also the current traffic density into choosing a route path, VANET messages, such as collision warning and cooperative driving, would be forwarded to destination much higher stability and reliability, nevertheless the network topology changes. When a relay node cannot route a message any more due to the topology dynamics, an alternative routing will greatly improve the message delivery rate. Similarly, letting the message stay on the junction to find out a new relay node will contribute to decrease the possibility of routing failure.

By comparing with GPSR, simulation results show that the proposed method improves on message delivery rate in the low vehicle density environment, while it takes much delay time than GPSR. However, in the high density environment, while the message delivery rate is kept high, the delay time is getting to similar with that of GPSR. For the future work, we are planning to study on improvement of performance related with delay time in low density environment. Moreover, we will consider a situation where message congestion occurs in intersection region heavily.

\section{ACKNOWLEDGMENT}

This work was supported in part by Mid-career Researcher Program through NRF grant funded by the MEST(No. 2009-0083985), and in part by the second stage of Brain Korea 21 project in 2010.

\section{REFERENCES}

[1] G. Korkmaz, E. Ekici, F. Ozguner, and U. Ozguner, "Urban multihop broadcast protocol for inter-vehicle communication system," Proc.1st ACM international workshop on Vehicular ad hoc networks, pp. 76-85, Oct. 2004.

[2] X. Yang, J. Liu, F. Zhao, and N. Vaidya, "A vehicle-to-vehicle communication protocol for cooperative collision warning," Proc. International Conference on Mobile and Ubiquitous Systems, pp. 114-123, Aug. 2004.

[3] J. Blum, A. Eskandarian, and L. Hoffman, "Challenges of intervehicle ad hoc networks," IEEE Trans. Intelligent Transportation Systems, vol. 5, no. 4, pp. 347-351, Dec. 2004.

[4] B. Karp and H. T. Kung, "GPSR: greedy perimeter stateless routing for wireless networks," Proc. ACM/IEEE Mobicom, pp. 243-254, Aug. 2000.

[5] Q. Li, and D. Rus, "Sending message to mobile users in disconnected ad-hoc wireless networks," Proc. ACM/IEEE Mobicom, pp. 44-55, Aug. 2000.
[6] J. Zhao and G. Cao, "VADD: vehicle-assisted data delivery in vehicular ad hoc networks," IEEE Trans. Vehicular Technology, vol. 57, no. 3, pp. 1910-1922, May 2008.

[7] W. Zhao, M. Ammar, and E. Zegura, "A message ferrying approach for data delivery in sparse mobile ad hoc networks," Proc. ACM Mobihoc, pp. 187-198, May 2004.

[8] Z. Mo, H. Zhu, K. Makki, and N. Pissinou, "MURU: a multi-hop routing protocol for urban vehicular ad hoc networks," Proc. 3rd Annual International Conference on Mobile and Ubiquitous Systems, pp. 1-8, Jul. 2006.

[9] S. Yu, H. Choi, and G. Cho, "A new recovery method for greedy routing protocols in high mobile vehicular communications," Proc. IEEE International Conference on Vehicular Electronics and Safety, pp. 45-50, Sep. 2008.

[10] D. Ahn, Y. Lee, and G. Cho, "A reliable message delivery method with considering traffic situation in VANET environment," Proc. International Conference on KIMICS, pp. 191-194, May 2010.

[11] D. Ahn, T. Kim, and G. Cho, "Handling reliable message delivery based on vehicle density in VANET routing," Proc. 6th International Conference on MITA, pp. 327-328, Aug. 2010.

[12] S. Olariu and M. C. Weigle, Vehicular Networks: From Theory to Practice, Chapman \& Hall, 2009.

[13] H. Liu, B. Zhang, H. Mouftah, X. Shen, and J. Ma, "Opportunistic routing for wireless ad hoc and sensor networks: present and future directions," IEEE Communications Magazine, vol. 47, no. 12, pp. 103-109, Dec. 2009.

[14] The Network Simulator, ns-2, http://www.isi.edu/nsnam/ns/

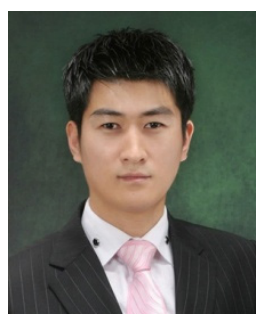

Do-Sik An received his B.S. and M.S. degree in computer science from Chonbuk National University, Jeonju, Korea, in 2008 and 2010. $\mathrm{He}$ is currently a Ph.D. student in Chonbuk National University, Korea. His current research interests are vehicular network, routing protocol, delay tolerant network, and bulk data transfer.

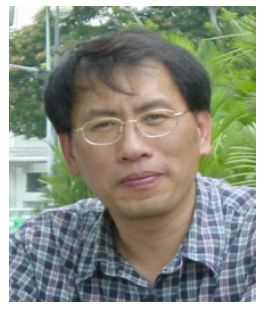

Gi-Hwan Cho received his B.S. degree from Chonnam University, Gwangju, Korea, in 1985, and the M.S. degree from Seoul National University, Seoul, Korea, in 1987, both in computer science and statistics. He received $\mathrm{Ph}$. D degree in computer science from University of Newcastle, Newcastle Upon Tyne, England, in 1996. He worked for ETRI(Electronics and Telecommunications Research Institute), Daejeon, Korea, as a Senior Member of Technical Staff from Sep. 1987 to Aug. 1997, for the Dept. of Computer Science at Mokpo National University, Mokpo, Korea, as a Full Time Lecture from Sep. 1997 to Feb. 1999. From Mar. 1999, he joined to the Division of Computer Science and Engineering at Chonbuk National University, Jeonju, Korea, and he is currently serving as a professor and chairman of the division. His current research interests include mobile computing, computer communication, security of wireless networks, sensor networks, and distributed computing system. 